\title{
Flow Characterization around Tandem Piers on Rigid Bed Channel
}

\author{
Laxmi Narayana PASUPULETI ${ }^{\bowtie}$, Prafulkumar Vasharambhai TIMBADIYA, \\ and Prem Lal PATEL
}

Sardar Vallabhbhai National Institute of Technology, Civil Engineering Department, Surat, India laxmiraagini@gmail.com

\begin{abstract}
The present study investigated flow characterization around tandem piers of circular shape having diameter $(d=8.8 \mathrm{~cm})$ at different radial angles, $\theta=0^{\circ}, 45^{\circ}, 90^{\circ}, 135^{\circ}$, and $180^{\circ}$ and compared with single pier of same diameter on rigid bed condition with identical flow conditions. The velocity fields at different depth around piers was measured using Acoustic Doppler Velocimeter (ADV). To characterize the flow, turbulence intensities, turbulence kinetic energy, and Reynolds shear stresses distributions are quantified and plotted for single and tandem piers. The Reynolds shear stresses are increased by $30 \%$ around the front pier and decreased by $30 \%$ around rear pier in tandem pier arrangement vis-à-vis single pier. In tandem case, turbulence intensity has shown 30\% increase in front pier and 30\% decrease in rear pier, as compared to the single pier. Further, in tandem arrangement, it has been found that there is significant decrease in the turbulence kinetic energy around the rear pier $(\approx 50 \%)$ vis-à-vis single pier.
\end{abstract}

Keywords: tandem arrangement, rigid bed, turbulence intensity, reynolds shear stress.

\section{INTRODUCTION}

The flow characterization around tandem piers are important to understand flow field dynamics. Several researchers attempted to study the turbulence around tandem piers in the past (AtaieAshtiani and Aslani-Kordkandi 2013; Laxmi Narayana et al. 2020). Further, Ataie-Ashtiani and Aslani-Kordkandi (2013) explored turbulence parameters around tandem piers on rigid bed and found that all these parameters are decreasing in tandem case in comparison to isolated case. However, the turbulence characteristics around the tandem piers at different radial angles $\left(\theta=0^{\circ}, 45^{\circ}, 90^{\circ}, 135^{\circ}\right.$, and $\left.180^{\circ}\right)$ was not studied in detail in past. This study is focussed on 
characteriation of turbulence fields around tandem bridge front and rear piers on rigid bed condition.

\section{EXPERIMENTATION AND METHODOLOGY}

The experiments around the single and tandem piers are performed using steel pipe as pier, having diameter $8.8 \mathrm{~cm}$ (Fig. 1). The pier is placed at predefined location and water was released into channel using SCADA system; a calibrated flow meter was fitted to inlet pipe to measure the water discharge. At $1 \mathrm{~m}$ upstream of the pier, at centreline of the flume, instantaneous 3D velocities were measured using ADV at several vertical positions starting from $0.5 \mathrm{~cm}$ above the bed at sampling frequency $40 \mathrm{~Hz}$ over a period of 180 seconds. The collected raw signals were post processed to eliminate the possible noise in the signals using phase space threshold technique proposed by Goring and Nikora (2002). The processed velocity signals were checked on Kolmogorov's scale.
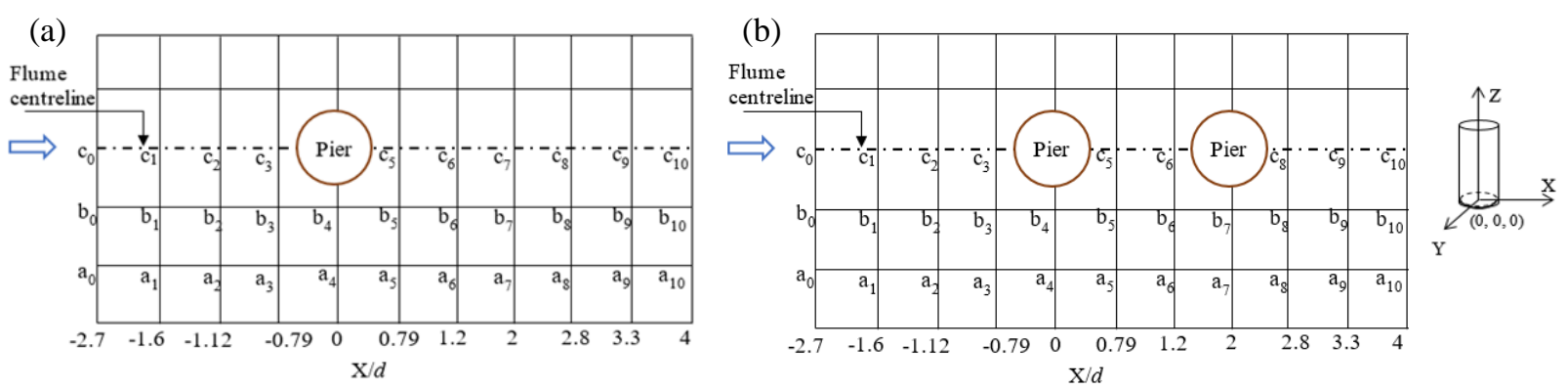

Fig. 1. Schematic of ADV data collection around the (a) single pier, and (b) tandem pier.

\section{RESULTS AND DISCUSSIONS}

The turbulence kinetic energy, turbulence intensity, and Reynolds shear stresses are computed as per studies of Ataie-Ashtiani and Aslani-Kordkandi (2013). The results revealed that the parameter has been found to increase around $\mathrm{X} / d=2$ in both configurations $(\mathrm{X}$ - distance from centre of pier in downstream, and $d$-diameter of the per). Further, higher turbulence kinetic energy has been found for single pier condition vis-à-vis tandem pier. Also, it is seen that, the parameter is maximum at $\theta=180^{\circ}(\theta$ is measured from positive $\mathrm{X}$-axis passing through centre of pier in anticlockwise direction).

The analysis of Reynolds shear stresses revealed that, as the flow approaches the pier, the component $\frac{-\overline{u^{\prime} w^{\prime}}}{U^{2}}$ is maximum at $\theta=0^{\circ}$ due to the downflow created just upstream of the pier. The Reynolds shear stresses have been found to decrease with increase in the angle with minimal value at $\theta=90^{\circ}$. The analysis also revealed that, shear stresses are $30 \%$ smaller in case of tandem pier vis-à-vis single pier condition.

The analysis of turbulence intensity revealed that, the parameter is increased with increase in the angle in both the cases. It was found that the turbulence intensities behind the single pier $\left(\theta=180^{\circ}\right)$, were strong, due to flow separation. Further, turbulence intensities are found to decrease by $30 \%$ behind the front pier in tandem pier condition vis-à-vis single pier condition at the same location.

Acknowledgments. Authors are thankful to Centre of Excellence (CoE) on "Water Resources and Flood Management" of SVNIT Surat funded under TEQIP-II, Ministry of Education (MoE), Government of India, necessary infrastructure support for conducting the experiments. 


\section{References}

Ataie-Ashtiani, B., and A. Aslani-Kordkandi (2013), Flow field around single and tandem piers, Flow Turbul. Combust. 90, 3, 471-490, DOI: 10.1007/s10494-012-9427-7.

Goring, D.G., and V.I. Nikora (2002), Despiking acoustic Doppler velocimeter data, J. Hydraul. Eng. 128, 1, 117-126, DOI: 10.1061/(ASCE)0733-9429(2002)128:1(117).

Laxmi Narayana, P., P.V. Timbadiya, and P.L. Patel (2020), Bed level variations around submerged tandem bridge piers in sand beds, ISH J. Hydraul. Eng., DOI: 10.1080/09715010.2020.1723138.

Received 22 March 2021

Accepted 12 April 2021 